\title{
LA FLORA INTESTINALE NEL PRIMO ANNO DI VITA DEL BAMBINO: PRIME CONSIDERAZIONI DALLO STUDIO ALLERGYFLORA.
}

Bonanno C.L.' , Benedetti C., Cocciolillo G.C.', Cristaldi A. ${ }^{2}$, Di Rienzo Businco A. ${ }^{2}$, Lauri S. ', Longo R. ', Mesiti A. ', Panetta V.', Spanò A. ', Tripodi S. ${ }^{3}$, Matricardi P.*

'U.O.C. Microbiologia e Virologia, ${ }^{2}$ U.O.C. Pediatria, ${ }^{3}$ U.O.S.D.

Allergologia Pediatrica - Ospedale "Sandro Pertini"- Roma;

* Asthma \& Allergy Research Unit

- Osp. Pediatrico Bambino Gesù - Roma

Introduzione. Il programma di ricerca Allergyflora della Comunità Europea si propone di valutare la relazione tra colonizzazione batterica intestinale nel primo anno di vita del bambino e sviluppo di allergie, in costante aumento negli ultimi decenni. Base del progetto è la Hygiene hypotesis del 1989, secondo la quale una stimolazione microbica appropriata è necessaria al sistema immunitario nell'infanzia per una corretta maturazione e funzione. L'aumento di allergie può essere dovuto, nei paesi a stile di vita occidentale, con meno agenti infettivi e maggiori misure igieniche durante il parto, ad una variazione della colonizzazione intestinale. Il progetto coinvolge tre centri europei (Svezia, Jnghilterra, Italia). L'acquisizione dati si è conclusa nel dicembre 2004. E' in corso l'analisi dei dati microbiologici contro vari fattori. Ne abbiamo qui considerati due: modalità di parto e sviluppo di eczema.

Metodi Sono stati arruolati 100 neonati per ogni centro. Di essi è stata studiata la flora aerobia a 3 giorni dalla nascita prelevandola con tampone rettale mentre la flora aerobia ed anaerobia è stata studiata con campioni di feci raccolti in anaerobiosi a 7,14, 28, 60, 180, 365 giorni e seminati in terreni standardizzati selettivi e non entro $24 \mathrm{~h}$.

Sono stati ricercati e quantificati Enterobatteri, Stafilococchi, Enterococchi, Lattobacilli, Bifidobatteri, Clostridi, Bacteroides e Lieviti (identificazioni con API 20E e Rapid Id32A Bio-Mèrieux).

Risultati A 3 giorni di vita il 50\% dei bambini italiani è colonizzato da Enterobatteri ed Enterococchi, il 40\% presenta Escherichia coli e solo il 5\% presenta Staphylococcus aureus. Ad una settimana di vita il $50-60 \%$ presenta clostridi, bifidobatteri e lattobacilli mentre bacteroides sono presenti nel $10-15 \%$. La conta degli aerobi tende a diminuire nel tempo mentre gli anaerobi sono quasi costanti. La colonizzazione varia secondo il parto: più aerobi a tre giorni nel parto vaginale, più anaerobi a 7 giorni nel cesareo (tranne Bacteroides).

Conclusioni Confrontando la flora microbica dei nostri soggetti (nove) che hanno presentato eczema nel primo anno di vita, i dati più significativi riguardano lo $S$. aureus (che negli atopici è presente a sei mesi in quasi 1'80\%) e Bacteroides, come studi svedesi avevano osservato.

Le nostre sono considerazioni preliminari: attendiamo ulteriori analisi epidemiologiche che ci confermino un reale nesso tra colonizzazione batterica ed allergie. 